\title{
Physical Simulation Experiment System of Multi-Rigid-Body Spacecraft Attitude Control Based on Micro-Gravity Floating Platform Environment
}

\author{
Yong-ming Gao ${ }^{1,}$, Zhao-ming $\mathrm{Li}^{1, \mathrm{~b}}$ \\ ${ }^{1}$ The Academy of Equipment \\ agym1701@163.com, bilovemolly@163.com
}

Keywords: multi-rigid-body spacecraft, flotation units, real-time simulation, virtual instrument

Abstract: This document mainly focuses on the problems existed in floating experiment such as software complexity, real-time difficulty and test method lackness. According to the simulation requirment, experiment of flotation units is proposed, hardware system of the simulation system is designed and software system is designed using virtual instrument technology. A variety of attitude maneuver modes are designed for the attitude control and simulation of multi-rigid-body physical prototype spacecraft. Experimental results indicate that the experimental system based on flotation units meets the requirements of multi-rigid-body spacecraft attitude control and simulation, and technology employed effectively solves the problems in the experiment.

\section{Introduction}

Multi-rigid-body spacecraft works in a complex space microgravity environment, an effective ground simulation is the key to ensure the success of spacecraft application in orbit. The attitude control of multi-rigid-body spacecraft plays a particularly important role in the mandate's successful completion, mainly including attitude stabilization and attitude maneuver. Generally, attitude control system consists of attitude sensors, controllers and actuators which indicate thrusters, magnetic moment and flywheel [1]. Complexity of the space task determines high agility and accuracy of attitude control, thus the general attitude actuators adopted are jet thrusters and flywheel.

The complexity of multi-rigid-body spacecraft system requires plenty of ground simulation test to verify and optimize the controller in the design and authentication process. Among them, full physical simulation is close to the final product as the simulation object, which is the most thorough simulation method in system development process. In addition to the attitude control simulation, it can also be used for the design of hardware and software verification. Full physical simulation has various experimental methods, gravity flotation experiment is the most important and feasible system. In order to successfully complete the task, it must ensure that the coordination of physical prototype service and security system, the main problems existing in the present experiment are:

1)Software's complexity and function integration's difficulty. If you use a general programming language, it is difficult to guarantee the software quality, unable to achieve fast and efficient integration.

2)Unable to guarantee the real-time. In the case of large amount of calculation, general operating system and a computing platform in the multi processor allocation and timing function deficiencies, can't effectively guarantee the real-time simulation. 
3)Single experimental model. In the float experiment, input data source can't meet the demand, many actions are unallowed to the physical simulation system, then the work state characteristic data can't adequately measure.

In the establishment of experimental system, some new technologies are adopted to solve these issues. Aim to the attitude control simulation system in this paper, firstly, functional analysis, according to the simulation demand, the experimental scheme are designed; then, set up the experimental system, the research focus on key technologies to develop the simulation software of virtual instrument and the PXI real-time platform; finally, select a number of attitude maneuver mode, simulate the physical prototype and MapleSim model of attitude control, and analysis the simulation data, or verify the MapleSim model.

\section{Flotation Platform experimental scheme}

The flotation experiments use real model in simulation, problems of actual components and control system can be found timely, to improve the reliability, to reduce the risk of out of control. In the experiment, the multi-rigid-body spacecraft environment is completely simulated by floatation platform, the use of compressed air with a layer of film is formed between the supporting air feet and platform, simulate the weightlessness of outer space, test the prototype by attitude maneuver or catching task. The experimental object is the physical prototype, control system adopts part or full of the physical components, the simulation software is also fully consistent with actual on-board software 0 . The experiment control the prototype by attitude control system, the onboard computer use PXI system, the main purpose of the experiment is the following four points:

Product design verification. The physical prototype is close to final products, the structure and the key components are basically consistent with the real, to solve the difficult how to establish accurate mathematical model, those shemes have the most powerful verification on hardware and function design.

Software design verification. The quality of simulation software determines the effectiveness. In order to improve the reliability of software, the flotation experiments are consistent with the actual satellite control software. In flotation experiments, the software key indicators are tested detailedly such as communication rate, stability, command response speed etc. through a variety of control mode, to find the problems in the software and correct them.

Parts performance test. The flotation experiments can take a variety of system or subsystem test, test whether the performance components meet the requirements, such as the flywheel torque accuracy, stability, failure rate and environmental adaptability, the measurement error of inertial measurement unit and so on.

Attitude control test. In physical simulation, the effects of the real parts of the control system performance can be intuitively and effectively reflected in the simulation results, which allow the user to detect the problem of control system design or physical parts of prototype. Attitude control system design specifications include stability, accuracy, overshoot, transition time etc, by the actual components running, high reliability can be tested and verified to optimize the control parameters. 


\section{Experimental system hardware design}

Experimental system components. Flotation units are generally divided into hardware platform simulation control systems, stage technology systems and ground support equipment [3]. Stage simulation control system is the important components, it includes three parts:

1)Attitude actuators. Including reaction wheels, jet thruster and the corresponding drive circuit;

2)Attitude measurement components. Including torque sensors, intersection cameras, hand-eye camera gyroscopes, accelerometers and so on;

3)Onboard computer. Including the PXI platform, various control cards and peripherals.

Flotation experiments use a sets of PID control algorithm based on attitude, onboard software integrates control, data acquisition, communication and other functions. The console is through wireless router to communicate with PXI, sending commands to the physical prototype simulation, while receiving state monitoring data and simulation data. The overall simulation system structure shown in Figure 1.

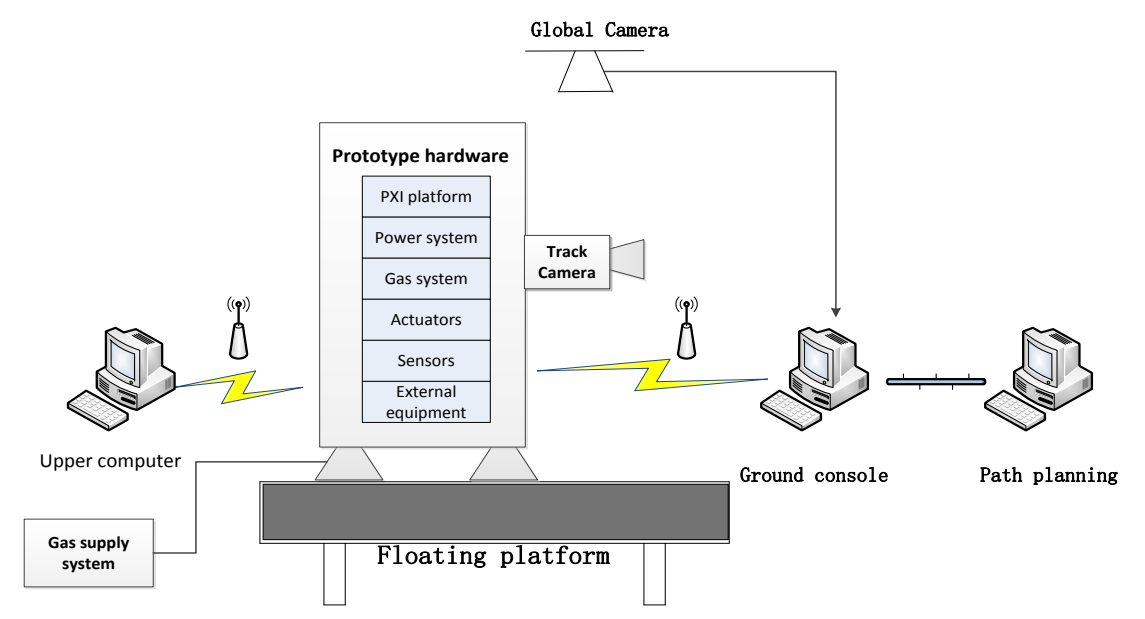

Figure 1 The structure of flotation simulation system

Prototype hardware design. To solve the problem of real-time simulation platform flotation system, using NI PXI platform as the onboard computer, the PXI platform designed specifically for real-time simulation based on CompactPCI standard, consists of the chassis, controller, I/O modules and peripheral equipment components. The PXI platform can be extended to hundreds of I/O channels, supporting all common bus protocols to meet the requirements of real-time simulation. During simulation, the Labview's RT module of the control program is transferred to the controller embedded processors automatically, runing in a dedicated real-time operating system, and complete a variety of simulation control functions through I/O modules sending and receiving data.

Physical prototype is mainly composed of the main frame, clapboard, PXI platform, power modules, gas supply systems, etc. The main frame is divided into three layers; the bottom placed the gas system; the middle place with weights; the top place PXI chassis, power supply systems, flywheels, inertial measurement unit, batteries, etc.; the jets are located at the side of the thrust of the middle frame. Computer uses the NI PXI platform and equipped with the relevant board. 


\section{Experimental System Software Design}

Flotation units are required to complete the simulation control simulation software, algorithms, data acquisition, state detection, cycle control and other functions, while the large number of hardware devices to communicate, so the use of virtual instrument technology is a better choice. NI Labview virtual instrument software, with graphical programming environment, strong instrument control and simulation of control, has been widely used in data acquisition and control, signal processing, data analysis and other fields [4]. Using Labview development tools, the development progress can be speeded up, flexible and quick to perform various mathematical simulation and semi-physical simulation.

Spaceborne simulation software architecture. Onboard software design. Onboard software development use virtual instrument software Labview, running on-board computer. It is necessary to onboard software perform complex control and communication functions, but also drive the hardware for data collection. Programming ideas must be used in the design of object-oriented, reasonable module division. Combination of hardware configuration and functional requirements, the software will be borne mainly divided into network communication module, attitude control module, inertial measurement module, torque sensor module, the intersection camera module. The attitude control module includes a flywheel driven, control algorithm computation, thruster control, mode selection function. Various functional modules run in the same VI in deployment, using multiple threads to realize.

Spaceborne simulation software technologies. The onboard modular software is achieved using of sub-VI, sub-VI is equal to function programming. Onboard software mainly has the following three key technologies:

1)Multi-thread design. Labview is an automated multithread language structure, which is decided based on the number of threads, distribution and switching. The thread execution system and priority can be configured directly in the properties dialog box of the VI. In the block diagram, an absolute loop or sub-VI can be a separate thread. Each module uses an absolute loop structure, the data transfer between the loop using the local variable, timing loops can also be used to set priorities and cycle settings.

2)Network communication. Labview provides a variety of network communication for users flexible choice, providing package VI function, which simplifies the programming. Program communication uses TCP/IP protocol, the center console as a server to listen on the specified port. Spaceborne program as a client sends a request to establish connection. The data package sender clusters, use the "flat into the string" for processing before sending, the data clusters will be revert. When sending, the first length of the string is sended, and then send the data string. 3)Serial communication. Serial communication adopts virtual instrument software architecture VISA(Virtual Instrument Software Architecture). This architecture is developed to improve efficiency by reducing the system time, so the independence of the $\mathrm{I} / \mathrm{O}$ interface is the principle of development. VISA can connect with most instrument, including GPIB, USB, serial port, PXI, VXI and Ethernet. VISA provides the unified programming interface through particular instrument driver. VISA provides advanced functions for serial communication, the detailed configuration on the serial port, such as timeout, baud rate, data bits and the parity check etc.

Onboard software of the host computer is loaded into the PXI real time system through the wireless network card. The host computer access and configure NI devices and systems through 
the MAX tool, communication with the terminal RT network system. The Real-Time module of Labview can effectively guarantee the real-time simulation.

\section{Simulation application of flotation Platform}

Simulation scheme design. In order to enrich the experimental mode, completing the test of system-level and component-level, a variety of attitude maneuver mode are used in experiment, for example, the attitude control mode for system-wide, angle maneuver mode for the flywheel, angle and displacement maneuver mode for thruster. The sufficient tests are taken for the attitude control system in various conditions.

Table 1 Attitude control mode description

\begin{tabular}{|c|c|c|c|}
\hline Worke mode & Model description & Pose determination method & Control mode \\
\hline Orbit maneuver & From one position to the desired position & The global camera & Thruster \\
\hline Orbit keep & Star base remains in a position & The global camera & Thruster \\
\hline Orbit maneuver & $\begin{array}{c}\text { From a attitude maneuver to desired } \\
\text { attitude }\end{array}$ & The global camera & Thruster \\
\hline Orbit maneuver & From a attitude maneuver to desired & The global camera & Freewheel \\
\hline
\end{tabular}

Simulation results analysis. During the flotation platform simulation, computer simulation is taken using the dynamic model [5]. The first, simulation results can be compared to prove the correctness of flotation platform; the second, adjusting the parameters of the attitude control system quickly; and then setting the parameters for the flotation experiments to reduce the risk of the experiment. The simulation model is created using MapleSim software, the parameters and properties are the same with physical prototype.

The first simulation step is the position approximation mode, which is simulated through thruster, the expectations data for the $\mathrm{X}$-axis is $0.2 \mathrm{~m}, \mathrm{Y}$-axis $0.3 \mathrm{~m}$. Displacement curve of flotation platform and MapleSim model are shown in Figure 2.

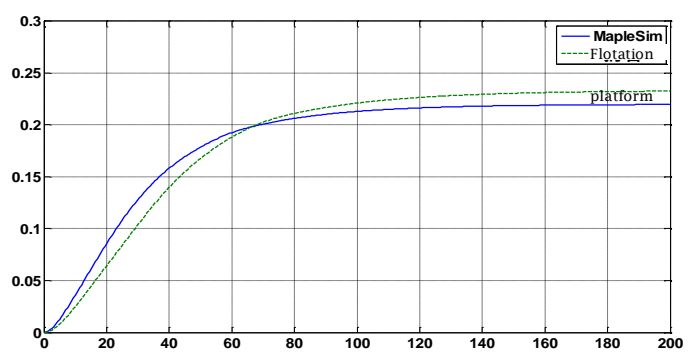

Displacement curves of $\mathrm{X}$ axis

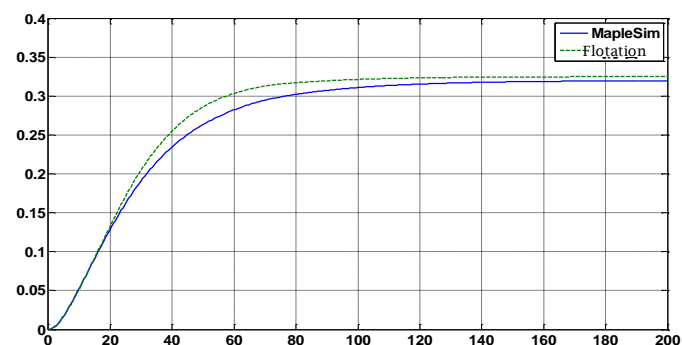

Displacement curves of $\mathrm{Z}$ axis

Figure 2 Experimental curve of position approximation

Then the second simulation step is the angle maneuver mode experiments uesing the flywheel to maneuver the base to the desired angle. Large scale angle and small angle maneuver are taken in experiments. The expectation of large scale angle is 2 radian, expectation of small angle is 0.5 radian. The curves of angle change are shown in Figure 3. 


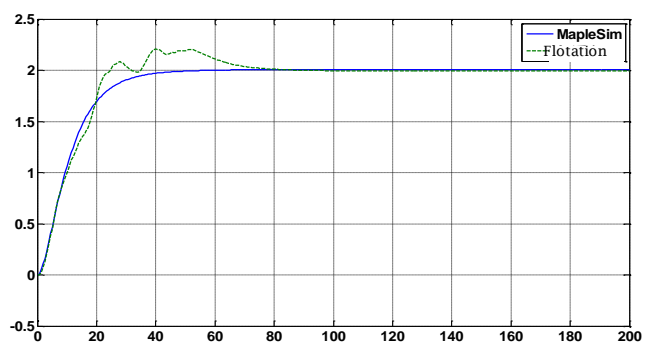

Curve of large angle maneuver

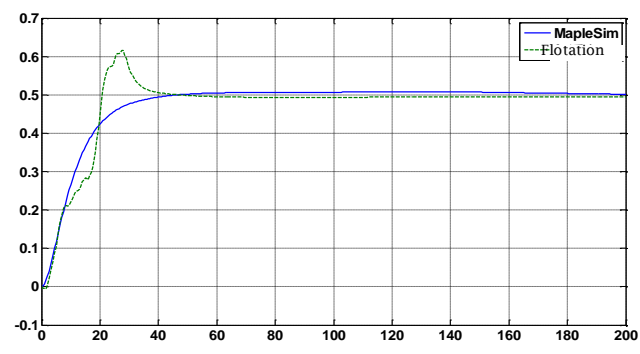

Curve of small angle maneuver

Figure 3 Experimental curve of angle maneuver

In the end, comparing the data of simulation results, we find that position is a little larger than expected in flotation platform experiment, there is a slight angle shock in angle maneuver experiment. Numerical variation tendency is consistent, the time to reach the maximum point is the same. Because the error of floation platform experiment is inevitable, then the experiment data can be considered valid, experimental model reflect the characteristics of the real system. Experiments also show that the use of virtual instrument technology can quickly combine the software and hardware equipment to complete the demands of real-time simulation. System uses PXI platform as the onboard computer; the Labview RT module automatically send the control program to embedded processor of the controller, running in the dedicated real-time operating system to ensure the real-time simulation.

\section{Summary}

The flotation experiments are the most close to the real environment, and the indispensable simulation means during prototype development stage. According to the simulation requirment, experiment of flotation units is proposed, hardware system of the simulation system is designed and software system is designed using virtual instrument technology. A variety of attitude maneuver modes are designed for the attitude control and simulation of multi-rigid-body physical prototype spacecraft. Experimental results indicate that the experimental system based on flotation units meets the requirements of multi-rigid-body spacecraft attitude control and simulation, and technology employed effectively solves the problems in the experiment. There is an important guiding significance for the ground test and verification of the multi-rigid-body spacecraft.

\section{References}

[1] PASTENA M, GRASSI M. Robust control of the smart microsatellite attitude dynamics[J]. Acta Astronautica, 2000, 47(2): 119-128.

[2] Li Jisu, Mou Xiaogang, Zhang Jinjiang. Application of Air Bearing Table in Satellite Control System Simulation [J]. Aerospace Control, 2008, 26(5): 64-68.

[3] Gao Huayu, Dong Yunfeng. Full--Physical Simulation Experiment System of Moonlet Attitude Control Based on Air Bearing Test--bed [J]. Computer Measurement\&Control, 2009(6): 1153-1156;

[4] Chen Xihui, Zhang Yinhong. Labview 8.20 Program Design from Rudiment to Conversance[M]. Tsinghua University Publish, 2007. 
[5] Jana L S. Mason A P, ChristOpher D H. Historical review of air-bearing spacecraft simulator[J]. Journal of Guidance, Control, and Dynamics, 2003, 26 (4): 513-522. 